\title{
Estudio cinético del sistema de molienda de una fábrica de cemento cubana
}

\section{Kinetic study of a grinding system in a cuban cement factory}

\author{
M. E. SALAS VINENT(*), A. C. RABILERO BOUZA ${ }^{(*)}$, V. CÓRDOBA RODRÍGUEZ ${ }^{(* *)}$ \\ (") CITMA (Ministerio de Ciencia, Tecnología y Medio Ambiente \\ (*) Filial Centro Técnica deDesarrollo de Materiales de la Construcción \\ (**) Facultad de Ingeniería Química Universidad de Oriente, \\ Santiago de Cuba
}

Fecha de aceptacion: 7-V-01

CUBA

\begin{abstract}
RESUMEN
Se presentan resultados obtenidos en el estudio cinético realizado al sistema de molienda de la fábrica de cemento de "Palmarito de Cauto", Santiago de Cuba, con vistas a la producción de cemento puzolánico PZ-250 con adición de hasta un $40 \%$ de puzolanas.

El presente trabajo avala satisfactoriamente las predicciones realizadas y permiten recomendar su utilización como un medio idóneo de eficiencia económica.
\end{abstract}

\section{SUMMARY}

A kinetic study realized in a moulding system in a cement factory ("Palmito de Cauto" Santiago de Cuba) is presented. This objective of this study is the production of a pozzolonic cement PZ-250 with an addition until $40 \%$ of pozzolan.

The present paper confirms the satisfactory predictions and permits to recommend the utilization it as a available way of economical efficiency.
PALABRAS CLAVE: puzolana, cinética, molienda

\section{INTRODUCCIÓN}

En Palmarito de Cauto (provincia de Santiago de Cuba), existe una planta diseñada y construida para la producción del aglomerante de cal-puzolana (ACP) conocido en nuestro país como cemento romano $(1,2)$. Esta planta cuya capacidad de producción de ACP de $100 \mathrm{kt} /$ año se ha visto limitada por no haberse construido los hornos de cal para su autoabastecimiento y depende de los excedentes de la calera ubicada en el CAI "Julio A. Mella" situado a unos $17 \mathrm{~km}$ de distancia; aunque se ha investigado con resultados positivos un yacimiento de caliza para cal situado a unos $2 \mathrm{~km}$ de la planta. La puzolana empleada es la conocida como toba manganeso (3-5) del yacimiento del mismo nombre, sobre el cual se encuentra
KEYWORDS: pozzolan, kinetic, grinding

edificada la planta. Esta puzolana es una toba zeolitizada (6-9) constituida por mordenita fundamentalmente y, en menor proporción, clinoptilolita y presencia variable de vidrio volcánico, así como otros minerales minoritarios.

No obstante, aunque el destino fundamental de la planta es la producción de ACP, ésta permite fabricar otros cementos $\mathrm{y} / \mathrm{o}$ aglomerantes gracias al equipamiento instalado y a la concepción del proceso tecnológico (Figura 1). La planta consta del siguiente equipamiento fundamental:

. Una instalación de trituración con un sistema CDM8CDM9 con triturador de mandíbulas y remoledor de cono en secuencia. 


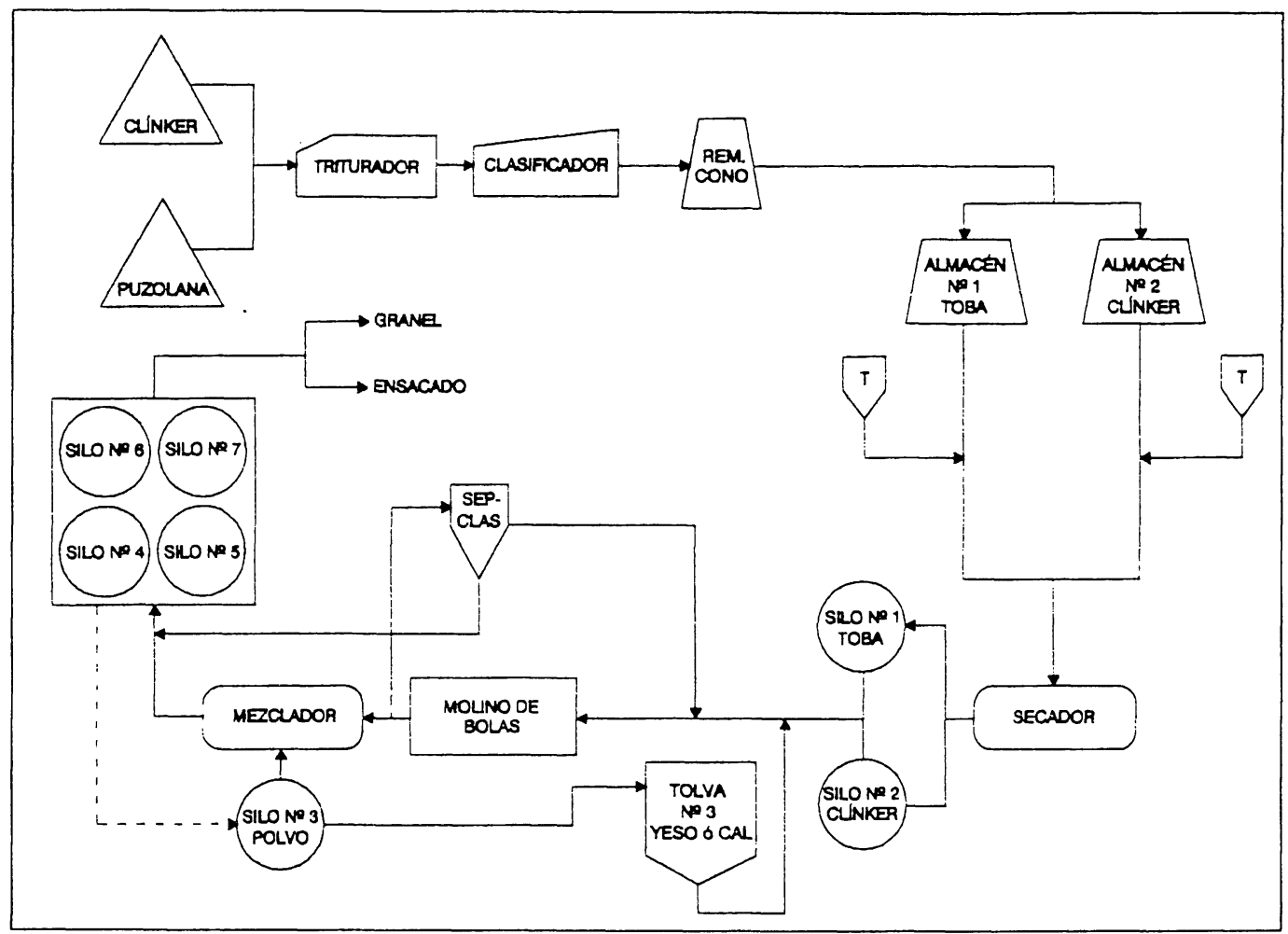

Figura 1.- Esquema del proceso tecnológico de producción de aglomerante de cal-puzolana de Palmarito de Cauto para la producción de elementos con adiciones, incluso cuaternarias.

. Un secador rotatorio con un generador de gases calientes que puede quemar indistintamente fuel-oil, inclusive crudo cubano, o gas-oíl, para producciones de zeolita de uso biológico.

. Un molino de bolas de una sola cámara procedente de una planta de cemento desactivada, el cual puede trabajar en circuito cerrado con un separador dinámico tipo Sturtevant de $8 \mathrm{t} / \mathrm{h}$, aunque, por diseño, falta por instalar un segundo separador para lograr la capacidad del sistema en la producción de ACP de 15 th $\mathrm{h}$.

\section{FUNDAMENTACIÓN TEÓRICA}

Los cementos puzolánicos de más de $20 \%$ y hasta $40 \%$ de a dición $(10,11)$ consisten en una mezcla intima y homogénea de la puzolana con el clínker más el regulador de fraguado, tanto por medio de la molienda conjunta como por la molienda separada y ulterior mezclado. Esto acarrea para las puzolanas naturales algunos problemas de ingeniería que deben ser resueltos: la trituración y el secado, así como la tecnología adecuada de molienda que implica, además, el control de la adición, lo cual requiere de un análisis de la teoría relacionada con la reducción de tamaño de las partículas sólidas.
La solución a los problemas anteriores depende de si se trata de una nueva planta por construir -por lo menos una nueva planta para asimilar las puzolanas en una planta ya existente- o si es necesario asumir la producción de cemento con adiciones de puzolanas en una planta existente sin modificar algunos equipos.

En los molinos de circuito abierto, las partículas pasan una sola vez a través del mismo, con un tiempo suficiente, de modo que se pueda obtener la finura deseada, la cual depende de las dimensiones del molino, del número de cámaras, de la abertura de las rejillas separadoras y de la carga de los cuerpos molturantes, así como también de la ventilación del molino, a lo que se añade el número de horas, granulometría del material y la temperatura alcanzada durante la molienda, que se ve incrementada sensiblemente por la del material, tal y como ocurre durante la molienda del clínker en la que la finura se ve afectada por la temperatura y el rendimiento horario del molino(12).

La calidad del cemento con adición puzolánica depende, en gran medida, de sus componentes: clínker, adición activa y regulador de fraguado $(13,14)$.

Obviamente, como la fabricación de un cemento de este tipo reside, fundamentalmente, en la molienda, ésta 
debe ser tal que permita aprovechar al máximo las características de los citados componentes, produciendo cementos que contribuyan a aumentar en las resistencias mecánicas, trabajabilidad y estabilidad de volumen en los hormigones y morteros en donde se empleen.

El efecto distribución granulométrica afecta, fundamentalmente, a la evolución de resistencias y a la trabajabilidad que pueda obtenerse de un cemento en morteros y en hormigones.

Para la obtención de las curvas granulométricas deseadas es evidente la importancia de seleccionar el tipo de molienda deseado -sistema de molienda- y, para ello, la aptitud a la molienda, es decir, la molturabilidad de cada materia prima es el parámetro más importante.

Los modernos sistemas de circuito cerrados de molienda con la inclusión de separadores de alta eficiencia permiten una mayor flexibilidad en el caso de molienda conjunta.

\section{RESULTADOS Y SU DISCUSIÓN}

A continuación se muestran los resultados del análisis del sistema de molienda de la planta con vistas a la producción de cemento puzolánico PZ- 250 con adición de hasta $40 \%$ de puzolana.

En este caso, el análisis del sistema de molienda ha sido realizado como si fuera a producirse cemento portland sin adición alguna (tan sólo clínker más yeso), pues la puzolana es más fácilmente molturable que el clínker y si los resultados son positivos para clínker + yeso, también lo serán, sin duda, para el caso de la molienda conjunta con las adiciones de puzolana.

\section{Características del posible clínker a moler}

Los análisis normales de granulometría del clínker, alimentado a los molinos de cemento, evidencian retenidos del $20 \%$ en los tamices de $10-20 \mathrm{~mm}$, muy altos para alimentar el sistema de molienda con un molino de una sola cámara, como es el caso de Palmarito.

Lo anterior implica la necesidad de trituración previa empleando el triturador de cono existente para lograr un retenido no superior al $20 \%$ en el tamiz de $5 \mathrm{~mm}$, así como lograr colapsar el mayor número de partículas.

Para facilitar la molienda deberá producirse un clínker con alto FSC y contenidos de cal libre no mayores del $3 \%$ y bajos valores de "peso/litro", en el orden de los 1.250-1.300 g/l, lo que ha de traducirse en menor consumo energético. Se ha tomado como índice de Bond para el clínker el valor de $14 \mathrm{kwh} / \mathrm{t}$.

\section{El sistema de molienda}

Como se ha indicado con anterioridad, el sistema demolienda está constituido por un molino de bolas de una sola cámara que puede trabajar en circuito cerrado con un separador tipo Sturtevant de $8 \mathrm{t} / \mathrm{h}$.

Las características del molino son las siguientes:

. Longitud (1)

. Diámetro interior $\left(\mathrm{D}_{\mathrm{i}}\right)$

. Volumen interior

. Potencia del motor

- Tensión

. Vel. de rotación ( $72 \%$ de la crítica) $22 \mathrm{r} / \mathrm{min}$

. Carga de bolas para $30 \%$ de llenado $37 \mathrm{t}$

\section{Cálculo del sistema de molienda}

Para la molienda de clínker más $4 \%$ de yeso solamente, con granulometría del clínker de $20 \%$ de retenido en el tamiz de $5 \mathrm{~mm}$ e índice de Bond de $14 \mathrm{kwh} / \mathrm{t}$, tenemos:

\section{Diámetro máximo de las bolas}

$$
\mathrm{d}_{\text {max }}^{2}=\frac{\mathrm{X}_{\mathrm{f}} \mathrm{W}}{\mathrm{NK}} \sqrt{\frac{\delta}{\mathrm{D}_{\mathrm{i}}}}(1)
$$

donde $\mathrm{X}_{\mathrm{f}}$ es la abertura del tamiz en el que se retiene el $20 \%$ del material alimentado, $\mathrm{W}$ es el índice de Bond, $\mathrm{N}$ el \% de la velocidad crítica de rotación, $\mathrm{K}$ es una constante para las bolas de acero e igual a 200, $\delta$ es la densidad del clínker y $D_{i}$ es el diámetro interior del molino en pies.

Entonces:

$\mathrm{d}_{\text {máx }}^{2}=\left(\frac{5.000 \mu \mathrm{m} \cdot 14 \mathrm{kWh} / \mathrm{t}}{72 \% \cdot 200}\right) \sqrt{\frac{3,15}{7 \mathrm{ft}}}=3,26$

Extrayendo la raíz cuadrada :

$\mathrm{d}_{\max }=1,81 \mathrm{in}=4,59 \mathrm{~cm}$; redondeando $\mathrm{d}_{\max }=50 \mathrm{~mm}$.

Para el 30\% de llenado ( 37 t) la distribución de carga ha de ser:

\begin{tabular}{|c|c|c|}
\hline Bola & de 5 & mmel $42 \% \ldots . .15,5 \mathrm{t}$ \\
\hline “ & 40 & el $42 \% \ldots \ldots . .15,5$ \\
\hline “ & 30 & $13 \% \ldots \ldots \ldots .4,8$ \\
\hline “ & 25 & 3\%.........1,2 \\
\hline & & $100 \% \ldots \ldots . .37,0$ \\
\hline
\end{tabular}




\section{Rendimiento del molino}

De acuerdo con la fórmula de Towarow:

$$
Q=q\left(\frac{a \cdot b \cdot c}{1.000}\right)\left(6,7 V \sqrt{\left.D_{i}\right)} \sqrt{\frac{G}{V}}\right.
$$

donde q es el caudal específico en $\mathrm{hk} / \mathrm{kwh}$ e igual a 30 para $10 \%$ de retenido en el tamiz de $90 \mu \mathrm{m}$, a es un coeficiente de molturabilidad igual a 0,9 para el clínker, b es un factor de corrección para molienda fina e igual a 1 para $10 \%$ de retenido en el tamiz de $90 \mu \mathrm{m}$; c es un factor de corrección para el tipo de molino, 0,8 para circuito abierto y 1,0 para cerrado por tener una sola cámara, $V$ es el volumen útil de molino igual a $28 \mathrm{~m}^{3}, \mathrm{D}_{\mathrm{i}}$ es el diámetro interior del molino y $\mathrm{G}$ es la carga de bolas, igual a $37 \mathrm{t}$.

Para circuito abierto, $\mathrm{Q}$ arrojó el valor de $6,8 \mathrm{t} / \mathrm{h}$, es decir, unas $7 \mathrm{t} / \mathrm{h}$, y para circuito cerrado $8,5 \mathrm{t} / \mathrm{h}$; aunque en este caso la capacidad del separador limitará el rendimiento hasta unas 6-7 t/h, siendo necesario señalar la necesidad de trabajar en circuito cerrado debido a que una sola cámara no puede garantizar en el caso del clínker que se alcance el grado de finura deseado de $10 \%$ retenido en el tamiz de 90 $\mu \mathrm{m}$; lo cual podrá ser apreciado en el análisis cinético.

\section{Análisis cinético del molino}

De acuerdo con nuestros trabajos realizados en molinos de cemento de tres cámaras en circuito abierto, en los cuales el proceso cinético de molienda es de primer orden, a partir de los valores de retenidos en el tamiz de referencia, diferentes puntos a lo largo del molino, arrojan valores del orden de $0,18 \mathrm{~m}^{-1}$ para la constante $\mathrm{K}$ de velocidad de molienda o conminución de la ecuación cinética de primer orden:

$$
\ln Q=-K l+\ln Q_{0}
$$

donde $Q$ es la fracción retenida en el tamiz de referencia, l es la longitud y $\mathrm{Q}_{0}$ el retenido en el material que se alimenta $(1=0)$.

Considerando $\mathrm{K}=0,18 \mathrm{~m}^{-1}$ y un retenido de $95 \%$ en el tamiz de $90 \mu \mathrm{m}$ y teniendo en cuenta la longitud interior libre del molino igual a 7,7 m obtendremos mediante la ecuación anterior:

$$
\ln Q=-\left(0,18 \mathrm{~m}^{-1} \times 7,7 \mathrm{~m}\right)+\ln 95=3,1678
$$

Entonces Q $=23,75$; es decir un retenido del $24 \%$ en el tamiz de $90 \mu \mathrm{m}$ a la descarga del molino (muy elevado) lo que implica el empleo del separador para retornar las partículas grandes al molino trabajando en circuito cerrado.

\section{El circuito cerrado}

En el balance de masas del sistema de molienda de circuito cerrado (Figura 2) obtenemos las siguientes ecuaciones:

A la entrada del molino:

$$
C R_{t}=(C-1) R_{g}+1 R_{0}
$$

En el separador:

$$
C R_{m}=(C-1) R_{g}+1 R_{f}
$$

donde $\mathrm{R}$ son los residuos sobre el tamiz de referencia $(90 \mu \mathrm{m})$ y $\mathrm{C}$ es el factor de circulación que indica el valor medio del número de veces que una partícula circula a través del sistema hasta alcanzar el tamaño deseado.

Entonces, considerando un factor de circulación de 1,4 (bastante bajo) debido a la capacidad del separador; tomando como residuos en el tamiz de $90 \mu \mathrm{m}$, para el producto final dispuesto por el separador $\left(\mathrm{R}_{\mathrm{r}}\right)$, el valor buscado del $10 \%$ $\mathrm{y}$, para el entregado a la salida del molino $\left(\mathrm{R}_{\mathrm{m}}\right)$, el valor del $24 \%$, obtenido del análisis cinético; se obtiene, para el material de retorno, desde el separador hacia el molino, nuevamente un residuo $\left(\mathrm{R}_{\mathrm{g}}\right)$ del $60 \%$, que se corresponde con lo aportado en la literatura para un sistema de estas características $(15,16)$.

Un resultado similar se obtiene a la entrada del molino, tomando como residuo en el tamiz de $90 \mu \mathrm{m}$ del material de alimentación $\left(\mathrm{R}_{0}\right)$, e $195 \%$ de antes, nos lleva a obtener, para el material de entrada al molino, trabajando en circuito cerrado, un residuo $\left(\mathrm{R}_{0}\right)$ del $85 \%$.

Aunque si se cuenta con un separador de mayor capacidad y/o eficiencia, el rendimiento del sistema de molienda puede alcanzar las $8,5 \mathrm{t} / \mathrm{h}$ y hasta algo superiores, dependiendo de las características del clínker, que para los cálculos presentes hemos considerado desventajosas; el sistema actual puede lograr la producción, con rendimientos de unas $6 \mathrm{t} / \mathrm{h}$ de cemento $\mathrm{PZ}-250$, con adiciones del orden del $35-40 \%$.

\section{VALORACIÓN ECONÓMICA}

Considerando una producción regular de cemento tipo PZ250 , con una adición de $35-40 \%$ de puzolanas, para un rendimiento del sistema de molienda de $6 \mathrm{t} / \mathrm{h}$, se pueden lograr los siguientes beneficios económicos:

Para tres turnos de trabajo de $8 \mathrm{~h}$ cada uno, se obtendrían $144 \mathrm{t} / \mathrm{d}$, lo que representaría en el año (365 días del año) $52.560 \mathrm{t} / \mathrm{a}$. El precio del cemento es de $25 \mathrm{USD} / \mathrm{t}$. 


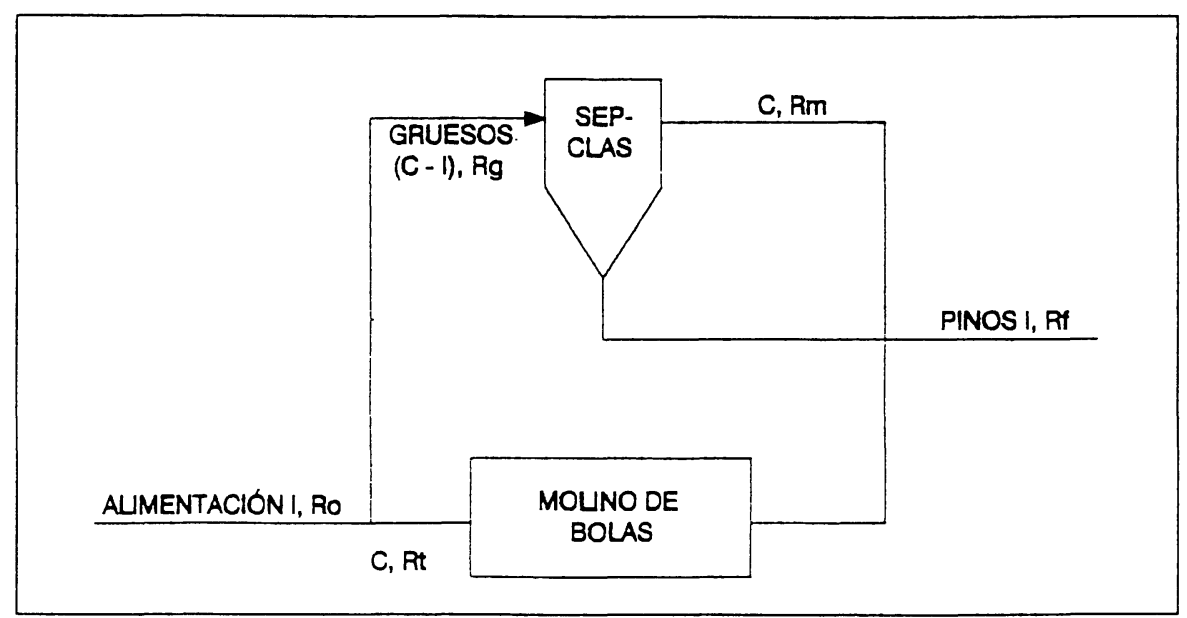

Figura 2.- Esquema de operación de molienda en un circuito cerrado, similar al de la planta de Palmarito de Cauto.

Se preve un ingreso bruto anual (IBA) de 1.314.000 USD/a. Teniendo en cuenta unos gastos de $15 \mathrm{USD} / \mathrm{t}$ por concepto de operación, mantenimiento, depreciación, salarios, transporte y otros; los gastos anuales (GA) serian de 788.400 USD/a.

Podrá lograrse entonces una ganancia neta anual (GNA) de:

$\mathrm{GNA}=\mathrm{IBA}-\mathrm{GA}$

$\mathrm{GNA}=525.600 \mathrm{USD} / \mathrm{a}$

\section{CONCLUSIONES}

De acuerdo con los resultados obtenidos y discutidos con anterioridad en el calculo cinético, así como su valoración económica, se pone en evidencia que el sistema de molienda de la planta de" Palmarito de Cauto", con adecuada carga de bolas y trabajando en circuito cerrado, puede, mediante molienda conjunta de clínker + yeso y puzolana, lograr la producción de cemento tipo PZ-250 con adiciones del $30-40 \%$ de puzolanas y un rendimiento de $6 \mathrm{t} / \mathrm{h}$.

\section{BIBLIOGRAFÍA}

(1) Velázquez, E. y Varona, O.(1987): "Tarea de proyección para la planta de cemento de Puzolana-Cal". IPROYAZ. Santiago de Cuba. Cuba.

(2) Velázquez, E., Rabilero, A . C. y Rosabal, J. C.(1994): "Planta para la producción de aglomerante puzolana-cal " IX Forum de Ciencia y Técnica, 6 p. La Habana. Cuba.

(3) Muñoz, J. A .( 1968): "Posibilidades de fabricación en Cuba de cementos puzolánicos. Universidad de Oriente. Cuba.

(4) Muñoz, J. A . y Rabilero, A . C. ( 1974): "Actividad puzolánica. Evaluación de una toba cubana". Revista Tecnológica. Vol. XII. No.5. pp 47-58.

(5) Muñoz, J. A . y Rabilero, A . C. ( 1978): "Cementos puzolánicos. Su fabricación en Cuba" , pp 7-34.

(6) Frye, K.(1985): The Encyclopedia of Mineralogy . Editorial Nedra, 512 p . Leningrado.

(7) Gorshkov, M. y Yakushova, A.( 1970): Geología General. Editorial Mir , 624 p. Moscú.

(8) Milovski, A. V. y Kónov.O.V .(1988): Mineralogía. Editorial Mir, 315 p. Moscú.

(9) Rizo, R. (1991): "Resultados de las investigaciones geólogo-tecnológicas de las zeolitas en las provincias orientales". III Conferencia Internacional sobre la presencia, propiedades y utilización de zeolitas naturales. La Habana. Cuba.

(10) Cuevas, Juan de las: "La industria cubana de los materiales de la construcción". MIMC. La Habana. 1993. 434 p.

(11) Rabilero, A. C.: "Contribución al empleo de puzolanas naturales en la producción de cementos y otros aglomerantes". Tesis de Doctorado en Ciencias Técnicas, Universidad de Oriente-CTDMC. 1996. 99 p.

(12) Nápoles, Ty Rabilero, A .C.: "Análisis de la finura del cemento portland en un sistema de circuito abierto." Trabajo de Diploma. Facultad de Ingeniería Química. ISPJAM. Santiago de Cuba. 1992.

(13) Herrero, E.: "Cementos con adiciones". Edición por el MIMC de los trabajos del autor empleados en el curso impartido en La Habana en $1990,118 \mathrm{p}$.

(14) Herrero, E. y J. R. Baragaño: "Cementos con adiciones activas". Cemento-Hormigón. No 652.1988. pp 179-217.

(15) Contreras, I., K. Matsushita y F.C. Pons (1989): Moagem e Moinhos, FILK; 301 p. Sao Paolo. Brasil.

(16) Duda, W. H.(1977): Manual Tecnológico del Cemento. Editores Técnicos Asociados, S.A,306 p. Barcelona. España. 